\title{
Management of Radiation Induced Xerostomia in Head and Neck Cancers
}

\author{
Rajeev Atri, Rakesh Dhankhar, Vimoj Nair, Vivek Kaushal
}

\section{ABSTRACT}

Head and neck squamous cell carcinoma is the most diverse class of malignancies lumped together under one diagnostic heading, occurring in several different sites and linked only by a common squamous histology. For cure in head and neck cancer dose of radiotherapy more than 60Gy are needed though the tolerance of normal organs e.g. salivary glands varies between 32Gy to 46Gy. Xerostomia is a major complication in patients who are receiving curative radiotherapy for head and neck cancer. Xerostomia is the main clinical effect that interferes with nutrition \& use of dentures, deteriorates oral hygiene and predisposes patients to oral candidiasis and dental problems (e.g. dental caries). In the worst case, dry mouth can lead to osteoradionecrosis of the mandible. Treatment of radiation induced xerostomia calls for good hydration, optimal oral hygiene and prophylaxis of candidiasis. Therapeutic options are artificial saliva substitutes, mouth wetting agents and drugs like pilocarpine and amifostine. Modern radiotherapeutic techniques requiring meticulous planning may lead to prevention of radiation induced xerostomia, however once xerostomia establishes the satisfactory measures of control are limited.

\section{Contact Author}

Dr. Rajeev Atri

E-mail : rajeevatri@gmail.com

Key Words: Xerostomia, Radiotherapy, Pilocarpine, Oral Hygiene.

$\mathrm{H}$ ead and neck cancers, among the 10 most frequent cancers in the world, constitute $5 \%$ of all cancers world wide.(1) In countries like India and China, cancers of the head and neck are one of the commonest cancers.(2) They accounts for one-fourth of male and one-tenth of female cancers in India.(3) HNSCC is the most diverse class of malignancies lumped together under one diagnostic heading, occurring in several different sites and linked only by a common squamous histology.(4) According to Gregory et al (1993), HNSCC comprises mainly squamous cell carcinoma of mucous membrane of upper aerodigestive tract.(4) Commonly seen sites are mucous membranes of oral cavity, oropharynx, larynx, nasopharynx, hypopharynx, cervical esophagus, nose, paranasal sinuses and lips.

Conventional radiotherapy has been shown to produce favorable results in early carcinomas of head and neck (T1 and T2 tumors) with cure rates of $80-90 \%$ and $50-70 \%$ for T1 and T2 tumors respectively. For the locally advanced carcinomas (T3 and T4 tumors), the local and regional control rate is extremely poor with three-year disease free survival of about $25-30 \% .(5-7)$

A definitive course of radiation therapy in head and neck carcinoma extends for about 6 weeks and is accompanied by its acute \& late reactions. The low-grade changes are called as reactions whereas severe forms of the same injury may be identified as toxicity.(8) The various radiation reactions frequently encountered during radical radiotherapy of head and neck cancers are as follows: radiation mucositis, skin reactions, xerostomia, dysgeusia, subcutaneous edema, alopecia osteonecrosis of mandible, severe dysphagia, laryngeal edema, dental decay etc. Xerostomia is one of the major complications of Radiotherapy. 


\section{Xerostomia}

Xerostomia is detected as a consequence of progressive impairment of salivary gland cells.(9) In xerostomia, or dry mouth, there is a subjective sensation of oral dryness that usually implies a marked decrease in salivary secretion. The serous acinar cells of parotid and submaxillary salivary glands undergo interphase death, and hence salivary dysfunction appears after irradiation, with no threshold dose and little sparing effect by fractionation. The magnitude of damage depends both on the volume of tissue irradiated and dose of radiation delivered.(10,11)

Changes in salivary glands are first indicators of radiation related damage. There is significant decrease in flow even after few fractions of radiotherapy.(1) Dryness of mouth thus is a common feature encountered during and after radiotherapy. The salivary secretion progressively decreases with increase in radiation dose beyond 50Gy.(1) The radiation side effects are because of imbalance between low radiation tolerance of the organ and the high tumor dose, which has to be delivered for cure. For cure in head and neck cancer doses more than $60 \mathrm{~Gy}$ are needed though the tolerance of normal organs e.g. salivary glands varies between $32 \mathrm{~Gy}$ to $46 \mathrm{~Gy}$.(9) Out of all the radiation reactions xerostomia is one of the commonest and most troublesome.

\section{Salivary Glands}

Parotid gland is histologically a serous type of gland and secretes $20 \%$ of total saliva. Submandibular salivary gland is histologically mixed type of gland and secretes $70 \%$ of total saliva. Sublingual salivary gland is histologically mixed type of gland and secretes 5\% of total saliva.(12) In salivary glands the secretory granules containing salivary enzymes are discharged from acinar cells into ducts. About $1500 \mathrm{ml}$ of saliva is secreted per day.

\section{$\mathrm{PH}$, Composition and control of salivary secretion}

The $\mathrm{pH}$ of saliva from resting gland is slightly less than 7, but during active secretion it approaches.(8) Saliva contains two digestive enzymes: Lingual lipase, secreted by glands on tongue, and ptyalin, secreted by salivary glands. Saliva also contains mucins, glycoproteins that lubricates the food and protect oral mucosa. It also contains $\operatorname{Ig} \mathrm{A}$, the first immunological defense against bacteria and viruses; lysozyme, which attack the wall of bacteria; lactoferrin, which binds iron and is bacteriostatic; and proline rich proteins that protect tooth enamel. There is considerable variation in ionic composition of saliva.

Salivary secretion is under neural control. Stimulation of parasympathetic nerve supply causes profuse secretion of watery saliva and relatively low content of organic matter. Associated with this secretion is pronounced vasodilatation of gland. Stimulation of sympathetic nerve supply causes vasoconstriction, secretion of small amount of saliva, rich in organic contents from submandibular glands. Food in mouth cause reflex secretion of saliva, and so does stimulation of vagal afferent fibers at gastric end of esophagus.(12)

Xerostomia may be an inconvenience when mild, or a debilitating condition when severe. The decreased salivary flow causes chronic oral discomfort and functional problems. Xerostomia is the main clinical effect that interferes with nutrition, deteriorates oral hygiene, and predisposes patients to oral candidiasis and dental problems (e.g. dental caries).(13) The symptom of dry mouth may not always correlate with the degree of diminished salivary flow, but a significant loss of salivary flow makes it difficult to process solid food into a bolus that can be swallowed. As a result, nutritional deficiencies may occur.(13) Since saliva also facilitates the formation of speech patterns; its loss hinders speaking and communicating, possibly causing the patient to withdraw from social interaction.(13) Together these conditions can impair the physiological and psychological well being of the patient.

The most frequently reported cause of xerostomia is the use xerostomic medications.(14) A number of commonly prescribed drugs with a variety of pharmacological activities have been found to produce xerostomia as a side effect. Additionally, xerostomia often is associated with Sjogren's syndrome; rheumatoid arthritis or a related connective tissue disease may accompany a condition that involves dry mouth and dry eyes. Xerostomia also is a frequent complication of radiation therapy. Complications of xerostomia include dental caries, candidiasis or difficulty with the use of dentures. A thorough intraoral and extra-oral clinical examination is important for diagnosis.(14)

Rydholm and associates conducted a study to explore the global effects of xerostomia, with a specific focus on psychological and social consequences.(15) Four main categories were identified in the study:

- Subjective discomfort, e.g. dryness or burning sensation,

- Loss of function, e.g. articulation or swallowing,

- Increased infection, (oral thrush and ulcerations),

- Psychosocial effects, including shame, increased feelings of being a patient rather than a person and a tendency to avoid social contact, resulting in loneliness.

Xerostomia and its associated symptoms have a considerable, negative global impact, resulting in shame, anxiety, disappointments and verbal communication difficulties. There should therefore be more focus on the management of xerostomia, which is often neglected in palliative care (15).

Dusek M et al conducted a study comparing masticatory performance and muscle activity of patients suffering from 
xerostomia with age \& sex, and number of occluding pairsmatched healthy controls. Masticatory function was evaluated by assessment of chewing motion and muscle activity during chewing an artificial food (CutterSil), chewing gum and swallowing a bolus of almond. Chewing motion was recorded with track computer system. Bilateral muscle activity of both masseter and anterior temporalis was recorded using surface electrodes. Results of this study revealed significant differences between patients and controls in their ability to process food and masticatory muscle activity. The majority of patients could not break down the artificial food, others had a larger median particle size than the controls. A significant difference was also observed in the number of chewing cycles required to swallow almonds, the patients required more than twice as many chews as the controls, $\mathrm{p}<0.001$. These findings suggest that patients with xerostomia exhibit reduced ability to process food. The observed decline in masticatory performance is probably due to reduced activity of the muscles of mastication.(16)

The assessment of severity of xerostomia is done by subjective and objective techniques. The Visual Analogue Scale, Zimmerman Xerostomia Questionnaire, LENT SOMA (Late Effect of Normal Tissues Subjective Objective Management Analysis) Scale are some of the methods to find and Grade the severity of xerostomia. The salivary gland secretory ratio (SGSR), determined by dynamic salivary $99 \mathrm{mTc}$ scintigraphy, is an objective measure of salivary gland function. $(17,18)$

\section{Management of Xerostomia}

Management of radiation induced xerostomia calls for good hydration, optimal oral hygiene and prophylaxis of candidiasis. Therapeutic options are artificial saliva substitutes, mouth wetting agents and drugs like pilocarpine and amifostine.(13) The first two provide short-term relief and may cause beneficial effects if no contraindications exist. Amifostine has demonstrated some efficacy in reducing xerostomia in patients of head and neck cancers receiving RT. Oral pilocarpine has received the approval of the U.S. Food and Drug Administration (FDA) for xerostomia induced by radiation therapy for head and neck cancers.(19) It is now being used in India for treatment of radiation-induced xerostomia. Conformal and Intensity Modulated RT are precision techniques for delivering exact dose dose to tumor volume sparing normal tissues.

\section{Preventive Methods}

Xerostomia occurs in approximately $75 \%$ of patients treated with conventional beam arrangements. Temporary xerostomia and loss of taste may last for several months. In patients receiving definitive conventional radiotherapy grade- 2 xerostomia is seen in $80 \%$ of patients while in only $30 \%$ of patients receiving focused radiation treatment like definitive Intensity Modulated Radiation Therapy (IMRT).
Conformal and Intensity Modulated RT are precision techniques for delivering exact dose dose to tumor volume sparing normal tissues. These are comparatively newer techniques specially for developing nations. Patients are planned with three-dimensional (3D) simulation and treated in the supine position, immobilized with a thermoplastic mask. IMRT is a very high-technology way of delivering radiation such that the amount of radiation given to the tumor can be maintained or even increased while actually decreasing (sometimes astonishingly so) the radiation dose received by normal tissues. This is done by combining a huge number (up to hundreds) of mini-beams (also called "segments") of varying radiation intensity, but all focused on part or all of the tumor (or an area suspected of containing tumor). IMRT has only been possible in recent years, the result of enormous advances in computer technology and in the mechanical hardware that administers radiation.(20)

The acute toxicity of irradiation assessed by Chao and colleagues in 430 patients with carcinoma of the oropharynx was comparable in five treatment groups. Late complications were also comparable except for late salivary toxicity, which was lower in the IMRT- treated patients. There was a significantly higher incidence of late xerostomia in patients receiving definitive conventional irradiation and postoperative conventional irradiation compared to those receiving definitive or postoperative IMRT $(\mathrm{P}<0.0001)$; IMRT can reduce this complication rate by $4 \%$ per Gy.(20)

Lin A et al compared Parotid-sparing intensity-modulated radiotherapy (IMRT) for head-and-neck cancer with standard RT to assess potential improvements in broader aspects of quality of life (QOL). They initiated a study of patient-reported QOL and its predictors after IMRT. This was a prospective longitudinal study of head-and-neck cancer patients receiving multisegmental static IMRT. Patients were given a validated xerostomia questionnaire (XQ), and a validated head-and-neck cancer-related QOL questionnaire. The questionnaires and measurements of salivary output from the major glands were completed before RT started (pre-RT) and at 3, 6, and 12 months after RT. Thirty-six patients participating in the study completed the questionnaires through 12 months. The study concluded that after parotid-sparing IMRT, a statistically significant correlation was noted between patient-reported xerostomia and each of the domains of QOL: Both xerostomia and QOL scores improved significantly over time during the first year after therapy. These results suggest that the efforts to improve xerostomia using IMRT may yield improvements in broad aspects of QOL.(21)

\section{Supportive Measures}

\section{Better oral hygiene}

As the physical stimulation of salivary glands originate in taste buds. The minimal, thickened, mucinous saliva produced by 
affected glands coats the mucosa of cheek tongue, palate. Similarly fungal infection forms the coating forming an effective barrier that prevents physical contact of taste buds by dietary substances and diminish physical response to thermal and mechanical stimuli. Mechanical debridment of dorsum of tongue with soft tooth brush and use of spray misters before and during meal assists in maintaining access to taste buds.

\section{Dental check-up}

Nieuw and associates recommended in a study that after the radiation therapy is ended, a dental check-up should be done every 3 months to allow control of any incipient oral inflammation and dental decay.(22) Fordailyuse, a special dentifrice (e.g. children's toothpaste) is recommended, since the taste of a regular dentifrice may be too strong for these patients. Different types of saliva substitutes are now commercially available, containing different polymers as thickening agents, e.g. arboxymethylcellulose (Oralube and Glandosane), polyacrylic acid, and xanthangum (Xialine).

\section{Antifungal prophylaxis}

Prevention of infection with antifungal prophylaxis may also help. Recent developments, which are, however, still in the experimental stage, are bioactive saliva substitutes and mouthwashes containing antimicrobial peptides to protect the oral tissues against microbial colonization and to suppress and to cure mucosal and gingival inflammation.

\section{Vitamin supplements}

Patients should be advised to maintain a balanced diet and avoid foods that irritate unprotected saliva Vitamin A and Nicotinic acid supplements are known to increase salivary flow.(23)

\section{Salivary substitutes}

There are many types of salivary substitutes available in the market. Salivary substitutes give only temporary relief like, Mucin containing sprays, Biotene, Sugar-free chewing gum, Lactoperoxidase, and glucose oxidase (Oral Balance) gel, Hydroxy-Propyl-Methyl-Cellulose (HPMC) etc. $(24,25)$

\section{Surgery}

Xerostomia is one of the permanent and devastating sequelae of head and neck irradiation, and its numerous consequences affect most aspects of a patient's life. Various surgical techniques like free jejunal graft, palatal reservoir in dentures and salivary gland transfer outside the radiation field have been used with varying amount of success

\section{Palatal reservoir}

Toljanic JA and Zucuskie T in a study recommended use of a palatal reservoir in denture patients with xerostomia. Dentures that included a reservoir for saliva substitute were constructed for three xerostomic patients. Results were obtained through examinations and interviews. All patients found that the reservoir system helped control the xerostomia.(26)

\section{Free jejunal patch graft}

Lam et al showed the advantage of using a free jejunal patch graft to restore large intra-oral mucosal defects and concurrently eradicate xerostomia. Further, it was recommended that a tracheostomy be used routinely in such cases to avoid postoperative aspiration of the graft secretions. Following healing the use of Osseo integrated implants can complete oral rehabilitation without compromising the graft.(27)

\section{Surgical transfer of submandibular salivary gland to submental space}

Jha et al hypothesized that the surgical transfer of submandibular salivary gland to submental space, outside the proposed radiation field, prior to starting radiation treatment, would prevent xerostomia. They conducted a prospective clinical trial on sixteen patients where the submandibular gland was transferred as part of the surgical intervention. The patients were followed clinically, with salivary flow studies and University of Washington quality of life questionnaire. All of the transferred salivary glands were positioned outside the proposed radiation fields and were functional. The patients did not complain of any xerostomia and developed only minimal oral mucositis. There were no surgical complications. Study concluded that surgical transfer of a submandibular salivary gland to the submental space (outside the radiation field) preserves its function and prevents the development of radiation-induced xerostomia.(28)

\section{Amifostine (Radio Protector)}

Radioprotectors are chemicals that reduce biological effect of radiation. The mechanism of action is scavenging of free radicals and restitution of free radical damage. Amifostine (WR-2721) is a phosphorothioate (prodrug) that is unreactive and penetrates poorly into cells till it is dephosphorylated by enzyme alkaline phosphatse to active metabolite WR-1065. Bone marrow, gut, liver, skin and salivary glands are well protected by amifostine. Brain and spinal cord are not protected. Amifostine is used in radiotherapy because it floods many normal tissues rapidly after administration but penetrates tumor very slowly. The strategy is to begin irradiation soon after administration of drug to exploit a differential effect. Toxicity profile includes nausea vomiting sneezing somnolence. Hypotension is the dose limiting toxicity.(29)

The US Food and Drug Administration (FDA) approved Amifostine for use in the setting of postoperative head and neck irradiation in June 1999. The Clinical Practice Guidelines of the American Society of Clinical Oncology state that the use of amifostine may be considered for reducing the incidence of acute and late xerostomia in patients receiving head and neck RT.(29) 


\section{Recombinant Human Keratinocyte Growth Factor (rHuKGEF)}

The use of compounds such as recombinant human keratinocyte growth factor (rHuKGEF) represents an approach to toxicity reduction differing from the classical radioprotection provided by amifostine. The concept is that the drug will promote and hasten the repair of treatment induced normal tissue damage. $\mathrm{rHuKGF}$ is a paracrine factor produced by measenchymal cells in epithelial tissues. A member of the fibroblast growth factor family (FGF7), it specifically stimulates epithelial cell proliferation and differentiation. In rodent models, rHuKGF administration increases the size and number of keratohyalin granules, increases overall epithelial thickness, and increases desmosomal attachments. When administered after RT in a pre clinical setting, a rapid increase in basal cell proliferation is seen. RT-and chemotherapyinduced injury to the oral and gastrointestinal tracts is significantly ameliorated with rHuKGF interaction when KGF receptor-positive squamous cell carcinoma is grown in the presence of $\operatorname{KGF}(30)$.

\section{Pilocarpine}

Pilocarpine is a cholinergic para-sympathomimetic drug exerting a wide range of pharmacological effects with predominant muscarinic action. Pilocarpine has been used for the alleviation of the symptoms of salivary gland hypofunction in patients with severe xerostomia following irradiation for head and neck cancer. The drug has also been used successfully for the prevention of radiation induced xerostomia in Head and Neck cancer patients. Pilocarpine produces a dose-related increase in salivary flow. It stimulates salivary secretion both in individuals with normal salivary gland function and in those with impaired salivary flow (xerostomia). Constituents of saliva- such as mucins, proteins, glycoproteins, and electrolytes are stimulated by pilocarpine. There are increases in salivary amylase lysozyme, total protein, and calcium after pilocarpine stimulation in normal subjects(31).

Most of the adverse events reported with pilocarpine are a consequence of exaggerated parasympathomimetic stimulation and are usually dose dependent, mild and self-limiting. However, severe adverse events might occasionally occur. In head and neck cancer patients, mild to moderate sweating, is the most prevalent side effect reported. Other adverse events reported are rhinitis, headache; increased urinary frequency, nausea, dizziness, vasodilatation, chills, dyspepsia asthenia, diarrhea, lacrimation, abdominal pain, vomiting, blurred vision, hypertension, constipation and abnormal vision. Side effects usually diminish within hours after cessation of therapy. Overdose should be treated with atropine titration $(0.5 \mathrm{mg}$ to $1.0 \mathrm{mg}$ given subcutaneously or intravenously). Pilocarpine is contraindicated in patients with clinically significant, uncontrolled cardio renal disease, uncontrolled asthma, known hypersensitivity to pilocarpine and when meiosis is undesirable such as in acute iritis, or when a decrease in the depth of the anterior chamber is undesirable as in narrow-angle (angle closure) glaucoma(31).

In head and neck cancer patients when given post RT, the recommended initial dose is one tablet of Pilocarpine three to four times daily. Tablets should be taken with a glass of water during or directly after meals with the last dose taken in conjunction with the evening meal. The maximum therapeutic effect is normally observed after 4 to 8 weeks of therapy. For patients who have not responded sufficiently after 4 weeks and are able to tolerate the dose of $5 \mathrm{mg}$ three times daily, a maximum dose of up to $30 \mathrm{mg}$ daily can be considered. Therapy should be discontinued if no improvement in xerostomia is noted after 2-3 months of therapy. There is no evidence suggesting the possibility of dosage adjustment in elderly patient population $(17,18)$.

In Head and neck cancer patients when given concomitant with RT tablet Pilocarpine (5mg) three to four times a day is usually started three days prior to RT and continued for three months. Safety and effectiveness has not been established in children. Zimmerman et al. published a retrospective study comparing head and neck cancer patients who were given pilocarpine concurrently with radiation therapy to patients who were not. This retrospective study looked at subjective xerostomia scores in a cohort of head and neck cancer patients who received pilocarpine during radiotherapy and three months afterwards, and similar cohort who received no pilocarpine. The pilocaroine treated group consisted of 22 consecutive patients whose radiation fields were likely to result in post treatment xerostomia. They were treated between September 1991 and June 1995. Pilocarpine Hydrochloride $5 \mathrm{mg}$ q.i.d. was administered beginning on first day of RT, continued for three months after completion of RT. The cohort who did not received pilocarpine consisted of 18 consecutively identified follow up patients who received radical RT, without receiving pilocarpine, for head and neck cancers. All patients had both parotids glands treated to dose of at least $45 \mathrm{~Gy}$. The comparisons were made by subjective assessments based upon Zimmerman Xerostomia questionnaire including oral dryness, oral comfort, ability to have uninterrupted sleep, ability to speak, and in eating.

This was identical to validated questionnaire used in twophase three clinical trials leading to FDA approval of drug. On this tool, subjective xerostomia was assessed utilizing a visual analogue scale measuring the severity of various components of dry mouth as mentioned. Responses consisted of single line drawn somewhere across the $10 \mathrm{~cm}$ scale. For each Group, the distance from the left side of scale was then measured in millimeters, averaged, and compared using the student t-test. All patients were treated with Cobalt or 4-6 MV photons. The majority of patients in both groups were 
treated for pharyngeal tumors. The mean interval between treatment and assessment of xerostomia was seventeen months. Average xerostomia score for patients who received pilocarpine was $68 \mathrm{~mm}$ and in group, which was not treated with pilocarpine, was $34 \mathrm{~mm}$. The pilocarpine group attained statistical superiority in all assessment comparisons individually and with the average of the combined assessments $(\mathrm{p}<0.01) .(17)$

Fox PC et al conducted a double-blind placebo-controlled trial to evaluate the efficacy of orally administered pilocarpine in treating oral dryness caused by salivary gland hypofunction. They studied the effect of pilocarpine on major salivary gland output and subjective responses in 31 patients with salivary hypofunction. Pilocarpine ( $5 \mathrm{mg}$ capsule, three times daily) was given for 5 months and a placebo was randomly for one month in a double blind fashion. Objective measurements of major salivary gland output which included measurements of salivary gland flow rates and salivary gland scintigraphy. Subjective impressions of oral moisture, treatment related side effects, number of psychological measures were assessed monthly. At low dosages, pilocarpine increased the production of saliva by parotid and submandibular and/or sublingual glands and relieved the sensation of oral dryness. The quantity and composition of pilocarpine-stimulated secretions were similar to saliva produced in response to gustatory stimulation with citrate. Pilocarpine is a safe, easily administered, effective therapy to relieve xerostomia by increasing natural salivary function.(32)

\section{Conclusion}

Radiation therapy to the head and neck region can be an effective form of treatment for malignancies. Unfortunately damage to salivary glands may occur. Xerostomia is the main clinical effect that interferes with nutrition \& use of dentures; deteriorates oral hygiene and predisposes patients to oral candidiasis and dental problems (e.g. dental caries). In the worst case, dry mouth can lead to osteoradionecrosis of the mandible. Xerostomia and its associated symptoms have a considerable, negative global impact, resulting in shame, anxiety, disappointments and verbal communication difficulties. The prevention and treatment of radiation induced xerostomia should be the primary concern and coordinated effort of a radiation oncologist and a dental surgeon now-adays.

Treatment of resultant dry mouth is at present poor. Therapeutic options are artificial saliva substitutes, mouth wetting agents and drugs like pilocarpine and amifostine. Modern radiotherapeutic techniques requiring meticulous planning may lead to prevention of radiation induced xerostomia, however once xerostomia establishes the satisfactory measures of control are limited.

\section{THE AUTHORS}

\section{Dr. Rajeev Atri}

M.D.

Senior Resident

Department of Radiotherapy, Regional Cancer Centre,

PGIMS, Rohtak, Haryana, India

E-mail-rajeevatri@gmail.com

\section{Dr. Rakesh Dhankhar}

M.D.

Assistant Professor

Department of Radiotherapy, Regional Cancer Centre, PGIMS, Rohtak,

Haryana, India

\section{Dr. Vimoj Nair}

M.B.B.S.

Junior Resident

Department of Radiotherapy, Regional Cancer Centre,

PGIMS, Rohtak, Haryana, India

\section{Dr. Vivek Kaushal}

\section{M.D., DNB}

Sr.Professor and Head

Department of Radiotherapy, Regional Cancer Centre,

PGIMS, Rohtak, Haryana, India

\section{References}

1. Mohanti BK, Bahadur S, Lal P, Gairola M, Rath GK. Cancers of Head and Neck. In: Rath GK, Mohanty BK, editors. Textbook of Radiation Oncology. 1st ed. New Delhi: B.I.Churchill Livingstone; 2000. p.131-43.

2. Tobias JS. Cancer of the head and neck. BMJ. 1994; 308:96174.

3. Yeole BB, Sankaranarayanan R, Sunny M Sc L, Swaminathan R, Parkin DM. Survival from head and neck cancer in Mumbai (Bombay), India. Cancer 2000; 89(2): 437-44.

4. Gregory TW, Scott ML, George EL, Waun KH. Head and neck cancer. In: James FH, Emil FN, Robert CB, Donald WK, Donald LM, Ralph RW, editors. Cancer Medicine. Philadelphia: Lea and Febiger; 1993.p.1211-74.

5. Million RR, Cassisi NJ, Mancuso AA. Oropharynx. In: Million RR, Cassisi NJ, editors. Management of head and neck cancer: a multidisciplinary approach. 2nd ed. Philadelphia: JB Lippinocott, 1994.p. 401-29. 30Perez CA, Carmichael T, Devineni VR, et al. Carcinoma of the tonsillar fossa: A nonrandomized comparison of irradiation alone or combined with surgery: Long-term results . Head Neck. 1991; 13: 282-90.

6. Fein DA, Lee WR, Amos WR, et al. Oropharyngeal carcinoma treated with radiotherapy: A 30-year experience. Int J Radiat Oncol Biol phys. 1996; 34: 289-96.

7. Jaulerry C, Rodriguez J, Brunin F, et al. Results of radiation therapy in carcinoma base of tongue. Cancer1991;67:1532.

8. Clliford Chao KS, Perez CA, Brady LW, editors. Radiation Oncology Management Decisions. 2nd ed. Philadelphia: Lippincott Williams \& Willkins; 2002. p.1-13.

9. Hall EJ, editor. Radiobiology for Radiologists. 5th ed. Lippincott Williams\& Willkins; 2000. p.339-60.

10. Leslie A, et al. Staging of squamous cell carcinoma of the oral cavity and oropharynx: a comparison of MRI and CT in T and Nstaging. J Comput Assist Tomogr. 1999; 23: 43-9.

11. Imola MJ. Technetium 99m single positron emission computed tomography scanning for assessing mandible invasion in oral cavity cancer. Laryngoscope. 2001; 111: 373-81.

12. Ganong WF. Regulation of gastrointestinal function. In: Ganong 
WF, editor. Review of medical physiology. $7^{\text {th }}$ ed. Connecticut: Appleton \& Lange; 1995.p. 260-72.

13. Berger AM, Kilroy TJ. Oral Complications. In: Devita VT, Hellman S, Rosenberg SA, editors. Cancer Principles and Practice of Oncology. 6th ed. Philadelphia: Lippincott-Raven; 2001. p.288192. 19.

14. Guggenheimer J, Moore PA. Xerostomia: Etiology, recognition and treatment. J Am Dent Assoc 2003; 134: 61-9.

15. Rydholm M, Strang P. Physical and psychosocial impact of xerostomia in palliative cancer care: A qualitative interview study. Int J Palliat Nurs. 2002; 8(7):318-23.

16. Dusek M, Simmons J, Buschang PH, Hashimi I. Masticatory function in patients with xerostomia. Gerodontology1996;13:3-8.

17. Zimmerman RP, Mark RJ, Juillard GF. Timing of pilocarpine treatment during head and neck radiotherapy: concomitant administration reduces xerostomia better than post-irradiation pilocarpine. Int J Radiat Oncol Biol Phys. 1996; 37 suppl 3. p.57175. 18.

18. Haddad P, Karimi M. A randomized double blind placebo controlled trial of concomitant pilocarpine with head and neck irradiation for prevention of radiation-induced xerostomia. Radiother Oncology. 2002; 64:29-32. 20.

19. Fisher J, Scott C, Scarantino CW, LeVeque FG, White RL, Rotman $\mathrm{M}$, et al. Phase III quality-of-life study results: impact on patient's quality of life to reduce xerostomia after radiotherapy for headand-neck cancer-RTOG 97-09. Int J Radiat Oncol Biol Phys 2003; 56.21.

20. Chao KSC, Majhail N, Huang CJ, et al. Intensity-modulated radiation therapy reduces late salivary toxicity without compromising tumor control in patients with oropharyngeal carcinoma: A comparison with conventional techniques, Radiother Oncol 2001; 49: 275-280.

21. Lin A, Kim HM, Terrell JE, Dawson LA, Ship JA, Eisbruch A. Quality of life after parotid-sparing IMRT for head-and-neck cancer: a prospective longitudinal study: Int J Radiat Oncol Biol Phys. 2003 Sep 1;57(1):61-70.
22. Nieuw Amerongen AV, Veerman EC. Current therapies for xerostomia and salivary gland hypofunction associated with cancer therapies. Support Care Cancer 2003;11:199-200.

23. Guchelaar HJ, Vermes A, Meerwaldt JH. Radiation induced xerostomia: Pathophysiology, clinical course and supportive treatment. Support Care Cancer. 1997; 5; 281- 8.

24. Warde P, Kroll B, O'Sullivan B. A phase II study of Biotene in the treatment of postradiation xerostomia in patients with head and neck cancer. Support Care Cancer. 2000; 8(3): 203-8.

25. Epstein JB, Emerton S, Le ND, Stevenson Moore P. A doubleblind crossover trial of Oral Balance gel and Biotene toothpaste versus placebo in patients with xerostomia following radiation therapy. Oral Oncol. 1999; 35(2): 132-7.

26. Toljanic JA, Zucuskie TG. Use of a palatal reservoir in denture patients with xerostomia. J Prosthet Dent. 1984; 52(4): 540-4.

27. Lam TC, Stevenson AR, Swift R. A cure for xerostomia? Free jejunal patch graft in oral reconstruction. Br J Oral Maxillofac Surg. 1995; 3:152-5.

28. Xerostomia M S, Jha N, Seikaly H, Harris J, Williams D, Liu R, McGaw T, Hofmann H, Robinson D, Hanson J, Barnaby P. Prevention of radiation induced xerostomia by surgical transfer of submandibular salivary gland into the submental space. Radiother Oncol. 2003; 66:283-9.

29. Brizel D, SauerR, strand V, et al. Randomized phase III trial of radiation \pm amifostine as a radioprotectant against xerostomia in head and neck cancer. J clin Oncol 2000; 18: 3339-45.

30. Ning S, Shuii C, Khan WB, et al. Effects of keratinocyte growth factor on the proliferation and radiation survival of human squamous cell carcinoma cell lines in vitro and in vivo. Int J Radiat Oncol Biol Phys 1997; 40:177-87.

31. Inged R, Faulds W, Faulds D. Oral pilocarpine: A review of its pharmacological properties and clinical potential in xerostomia. Drugs 1995; 1: 143-5.

32. Fox PC, van der Ven PF, Baum BJ, Mandel ID. Pilocarpine for the treatment of xerostomia associated with salivary gland dysfunction. Oral Surg Oral Med Oral Pathol 1986; 61(3): 243-8. 